\title{
Passivity Filter for Variable Impedance Control
}

\author{
Maciej Bednarczyk ${ }^{1}$, Hassan Omran ${ }^{1}$ and Bernard Bayle ${ }^{1}$
}

\begin{abstract}
While impedance control is one of the most commonly used strategies for robot interaction control, variable impedance control is a more recent preoccupation. If designing impedance control with varying parameters allows increasing the system flexibility and dexterity, it is still a challenging issue, as it may result in a loss of passivity of the control system. This has an important impact on the stability and therefore on the safety of the interaction. In this paper, we propose methods to design passivity filters that guarantee passivity of the interaction. They aim at either checking whether a desired impedance profile is passive, or modifying it if required.
\end{abstract}

\section{INTRODUCTION}

Robots are increasingly present in the same space as humans. The development of collaborative robots designed to safely support operators during the execution of tasks has certainly played an important role in this evolution, with applications such as lifting of heavy tools and pieces, hand-guiding or collaborative assembly. In this context, the question of handling physical contact in the best possible way has become crucial. This can be obtained typically by controlling the compliance of the interaction between the robot and its environment, resulting in the classical impedance control (IC) scheme [1]. IC consists in imposing a mass-spring-damper behavior to the whole system, for attracting the robot to the desired trajectory and for managing the interactions, both with the environment and potential humans involved in a collaborative task.

One of the main challenges in interaction management using IC lies in the proper selection of the impedance parameters, not only according to the task but also to ensure stability for all possible parameters variations. Indeed, if using fixed impedance parameters makes the system passive and hence stable when interacting with a passive environment [2], [3], this property no longer holds for arbitrarily varying parameters [4]. Not being able to modify the system impedance during the task is a notable drawback that may limit application ranges. For instance, human-robot interaction may benefit from the ability to adapt to human's force or fatigue, or to dynamically modify the interaction [5].

As a result, variable impedance control (VIC) has recently attracted more and more attention. VIC has been explored for challenging objectives, for example to deal with explosive movements [6], to optimize the performance of hammering tasks [7], allow risk-sensitive interactions [8] or maximize robot links velocities [9]. VIC has been implemented using reinforcement learning [10], as well as adaptive approaches for human-robot collaboration based on the estimation of the

\footnotetext{
${ }^{1}$ ICube, UMR 7357, University of Strasbourg, CNRS, 1 place de l'Hôpital, 67091, Strasbourg, France. m.bednarczyk@unistra.fr
}

human arm stiffness, from the derivatives of force and position [11], or from the measurement of muscle activity with electromyography [12]. However, in all these contributions, the resulting impedance of the robot is identified or learned during task execution, and therefore these methods cannot be used to check before execution whether a given impedance profile will lead to for stable execution.

Though in practice varying the stiffness does not necessarily lead to unstable behavior, several contributions addressed the issue of guaranteeing a stable execution of VIC, with a given impedance profile. In [13], the authors showed that exploiting kinematic redundancies can ensure passivity for a larger panel of impedance profiles. Gain scheduling control has also been used to address VIC by interpolating feedback gains between operating points [14]. Virtual energy-storing tanks [15] are another solution to modify the impedance model and guarantee passivity, by storing energy dissipated by the system. This stored energy can then be re-injected to simultaneously implement stiffness variations and in guarantee stable execution. This approach has been successfully implemented in several applications, as in [16], [17], or [18]. In [15], it is used to deal with the interactions resulting from tissues and membranes penetration during a robotic needle insertion.The tank-based strategy has been shown very well suited for VIC, in spite of some difficulties to tune its parameters. Nevertheless, it is dependent on the states of the system, measured during task execution and so can only be applied online.

Other authors proposed an approach that can be used to check the passivity of a given time-varying impedance profile before the execution of the task [4]. While this allows to check whether the interaction will have passive dynamics, it provides no solution to modify a profile that would not respect passivity conditions. The present paper aims to overcome this issue. Based on the combination of passivity conditions with an adaptation law on the impedance profile, the proposed solution allows checking whether a given profile is passive, and if it is not, it provides a method to modify it in a way to guarantee passivity. The proposed method can thus be used either offline, before the execution of the profile, or online.

The paper is organized as follows. Firstly, the control background and passivity analysis are introduced in Section II. Then, Section III describes the proposed design methods for passivity filters for VIC. In Section IV the features of the proposed passivity filters are explored, first in simulation and then using an experimental setup. Section $V$ finally provides conclusions and perspectives of the work. 


\section{CONTROL BACKGROUND}

\section{A. Rigid Body Dynamics and Linearized Model}

Consider the model of a $n$-joint serial robotic manipulator

$$
H(q) \ddot{q}+c(\dot{q}, q)+g(q)=\tau_{c}-J(q)^{T} f_{\text {ext }}
$$

where $\ddot{q}, \dot{q}, q \in \mathbb{R}^{n}$ are joint accelerations, velocities and positions, respectively, $\tau_{c} \in \mathbb{R}^{n}$ are the commanded joint torques, $f_{\text {ext }} \in \mathbb{R}^{m}$ is the end-effector wrench, $J(q) \in \mathbb{R}^{m \times n}$ is the robot Jacobian matrix and $H(q) \in \mathbb{R}^{n \times n}, c(\dot{q}, q) \in \mathbb{R}^{n}$ and $g(q) \in \mathbb{R}^{n}$ are the inertia matrix and the Coriolis and gravity terms, respectively. A classical solution to linearize equation (1) is to apply the computed torque control law, resulting in a double integrator model in joint space, or its equivalent in task space [19].

\section{B. Impedance Control}

In order to dynamically link the system positions, velocities and accelerations with the external forces, the input of the linearized system is computed in order to obtain

$$
M \ddot{e}_{p}+D \dot{e}_{p}+K e_{p}=f_{e x t}
$$

with $e_{p}=p_{r}-p \in \mathbb{R}^{m}$, where $p$ is the robot end effector pose and $p_{r}$ a reference motion. The interaction is then characterized by the impedance resulting from the apparent virtual mass $M \in \mathbb{R}^{m \times m}$, the desired damping $D \in \mathbb{R}^{m \times m}$ and the desired stiffness $K \in \mathbb{R}^{m \times m}$. Note that $M, D$ and $K$ are symmetric positive definite matrices and are chosen diagonal to simplify developments. For VIC, the case where $D(t)$ and $K(t)$ are time-varying is considered, while $M$ is assumed to be constant. From now on, time dependency will generally not be mentioned in the notations for simplicity.

\section{Passivity analysis}

In order to investigate the stability of VIC given by (2) with varying stiffness and damping terms, the following storage function can be considered

$$
V_{1}=\frac{1}{2} \dot{e}_{p}^{T} M \dot{e}_{p}+\frac{1}{2} e_{p}^{T} K e_{p}
$$

As $K$ and $M$ are symmetric, differentiating $V_{1}$ leads to

$$
\dot{V}_{1}=\dot{e}_{p}^{T} f_{e x t}+\left[\frac{1}{2} e_{p}^{T} \dot{K} e_{p}-\dot{e}_{p}^{T} D \dot{e}_{p}\right]
$$

If stiffness is constant or decreasing $\dot{V}_{1} \leq \dot{e}_{p}^{T} f_{e x t}$, which leads to the passivity condition

$$
V_{1}(t)-V_{1}(0) \leq \int_{0}^{t} \dot{e}_{p}^{T} f_{e x t} d \tau
$$

Because of stiffness time-dependency in equation (4), the sign of the term in brackets may change, possibly leading to a violation of the passivity condition (5) in case of increasing stiffness. Passivity can only be guaranteed if the stiffness is either constant or decreasing. In this case, the storage function can be used as a Lyapunov-like function to show stability. Also, it is well known that the interaction with a passive environment yields a passive, thus stable, interaction.

The storage candidate function (3) is conservative and should not be used to conclude on system passivity for increasing stiffness. Alternative storage functions need to be explored. In order to facilitate the passivity analysis, the authors of [20] propose to use a filtered tracking error-like variable $r \in \mathbb{R}^{m}$

$$
r=\dot{e}_{p}+\alpha e_{p}
$$

where $\alpha \in \mathbb{R}^{+}$is a constant. As shown in [21], if $r$ is bounded then $e_{p}, \dot{e}_{p}$ are bounded and, in this case, if $r \rightarrow 0$, then $e_{p}, \dot{e}_{p} \rightarrow 0$. With this in mind, it is possible to reformulate the control objectives using $r$

$$
\begin{aligned}
& \int_{0}^{t} r^{T} f_{e x t} d \tau=\int_{0}^{t}\left\{r^{T}\left(M \ddot{e}_{p}+D \dot{e}_{p}+K e_{p}\right)\right\} d \tau \\
= & \int_{0}^{t}\left\{r^{T}\left(M\left(\ddot{e}_{p}+\alpha \dot{e}_{p}\right)-\alpha M \dot{e}_{p}+D \dot{e}_{p}+K e_{p}\right)\right\} d \tau
\end{aligned}
$$

Using equation (6) and its derivative, it follows that

$$
\begin{aligned}
& \int_{0}^{t} r^{T} f_{e x t} d \tau=\int_{0}^{t}\left\{r^{T} M \dot{r}+\dot{e}_{p}^{T}(D-\alpha M) \dot{e}_{p}\right. \\
& \left.\left.\quad+e_{p}^{T}\left(K+\alpha D-\alpha^{2} M\right) \dot{e}_{p}+e_{p}^{T}(\alpha K) e_{p}\right)\right\} d \tau
\end{aligned}
$$

By introducing a symmetric, positive semidefinite and continuously differentiable matrix $C$, equation (7) can be written

$$
\begin{array}{r}
\int_{0}^{t} r^{T} f_{\text {ext }} d \tau=\int_{0}^{t}\left\{r^{T} M \dot{r}+e_{p}^{T} C \dot{e}_{p}+\frac{1}{2} e_{p}^{T} \dot{C} e_{p}\right\} d \tau \\
+\int_{0}^{t}\left\{\dot{e}_{p}^{T}(D-\alpha M) \dot{e}_{p}+e_{p}^{T}\left(\alpha K-\frac{1}{2} \dot{C}\right) e_{p}\right. \\
\left.+e_{p}^{T}\left(K+\alpha D-\alpha^{2} M-C\right) \dot{e}_{p}\right\} d \tau
\end{array}
$$

which can be written as

$$
\int_{0}^{t} r^{T} f_{\text {ext }} d \tau=V_{2}(t)-V_{2}(0)+\int_{0}^{t} W_{2} d \tau
$$

with

$$
V_{2}=\frac{1}{2} r^{T} M r+\frac{1}{2} e_{p}^{T} C e_{p}
$$

and

$$
\begin{array}{r}
W_{2}=\dot{e}_{p}^{T}(D-\alpha M) \dot{e}_{p}+e_{p}^{T}\left(\alpha K-\frac{1}{2} \dot{C}\right) e_{p} \\
+e_{p}^{T}\left(K+\alpha D-\alpha^{2} M-C\right) \dot{e}_{p}
\end{array}
$$

It is worth noticing that if we define $C=K+\alpha D-\alpha^{2} M$, the candidate storage function (10) is the same as in [4], resulting in the stability the conditions

$$
\begin{aligned}
& 0 \leq D-\alpha M \\
& 0 \leq 2 \alpha K-\dot{K}-\alpha \dot{D}
\end{aligned}
$$

This particular choice leads to $W_{2} \geq 0$, allowing to conclude on system passivity, as in this case equation (9) leads to

$$
V_{2}(t)-V_{2}(0) \leq \int_{0}^{t} r^{T} f_{e x t} d \tau
$$

Passivity conditions (12) and (13) can be used in order to verify whether passivity is guaranteed for a given impedance profile. However, they do not give any information about how to modify a profile that is not passive. In the following, we will be interested in designing a method capable of guaranteeing passivity properties for any given impedance profile based on conditions (12) and (13). 


\section{PASSIVITY FILTERS FOR VIC}

This section introduces the design of a passivity filter, that makes it possible to modify a non passive impedance profile online, in order to guarantee passivity.

\section{A. Guaranteeing passivity}

Let us consider the change in system impedance, with the stiffness (respectively the damping) varying from $K_{0}$ to $K_{1}$ (respectively from $D_{0}$ to $D_{1}$ ). In the following only the case $K_{0}<K_{1}$ is considered, as stiffness increase is the only case for which the passivity condition can be violated, as stated in Section II-C. Let us now define $K$ and $D$ such that

$$
\begin{gathered}
K=K(t)=K_{0}+\Gamma(t) \delta K \\
D=D(t)=D_{0}+\Gamma(t) \delta D
\end{gathered}
$$

with $\delta K=K_{1}-K_{0}, \delta D=D_{1}-D_{0}$ and $\Gamma$ a diagonal matrix where all diagonal terms $\gamma_{i}$ are differentiable gains such that $0 \leq \gamma_{i} \leq 1$.

As $M, K, D$ were chosen to be diagonal, the impedance behavior can be decoupled. In order to ensure passivity using conditions on $V_{2}, K$ and $D$ need to satisfy equations (12) and (13). Condition (12) allows choosing $\alpha$ easily. For instance, in [4] it is chosen as the ratio between the smallest eigenvalue of $D$ and the largest eigenvalue of $M$. Condition (13) implies that $\forall i \in\{1,2, \ldots, n\}$

$$
\dot{\gamma}_{i}\left(\alpha \delta d_{i}+\delta k_{i}\right)-2 \alpha\left(k_{0 i}+\gamma \delta k_{i}\right) \leq 0,
$$

with $\delta k_{i}$ and $\delta d_{i}$ the diagonal terms of $\delta K$ and $\delta D$ respectively, and $k_{0 i}$ the diagonal terms of $K_{0}$.

Generally, an increase in the desired stiffness does not come with a decrease in the desired damping, which would be the most constraining case in terms of stability. Then, let us assume that $\delta d_{i} \geq 0$. As $\delta k_{i}>0$ and $\alpha \geq 0$, then $\alpha \delta d_{i}+\delta k_{i}>0$. Equation (17) can then be written as

$$
\dot{\gamma}_{i} \leq a_{i} \gamma_{i}+b_{i}
$$

with

$$
a_{i}=\frac{2 \alpha \delta k_{i}}{\alpha \delta d_{i}+\delta k_{i}} \quad \text { and } \quad b_{i}=\frac{2 \alpha k_{0 i}}{\alpha \delta d_{i}+\delta k_{i}}
$$

Combining equation (17) with a low-pass filter, in order to ensure smoothness of $\gamma_{i}$, a passivity filter can be designed in such a way that it takes as input the desired switching profile $\bar{\gamma}_{i}$ and generates an output profile $\gamma_{i}$ that guarantees the system passivity. The filter can be then defined by

$$
\dot{\gamma}_{i}=\min \left(\beta\left(\bar{\gamma}_{i}-\gamma_{i}\right), a_{i} \gamma_{i}+b_{i}\right)
$$

where $\beta$ is the filter parameter. The integration of (19) permits to obtain $\gamma_{i}$ that tracks $\bar{\gamma}_{i}$ in such a way that passivity conditions are respected. Note also that if the damping decreases while the stiffness increases, the previous reasoning can be applied, but should be adapted, based on the numerical values of $\delta d_{i}$ and $\delta k_{i}$ (the case $\alpha \delta d_{i}+\delta k_{i}=0$ is singular and should be avoided).

Though the usage of the proposed filter may guarantee passivity, the tracking performance highly depends on the conservatism of the passivity conditions. The constant value $\alpha$ plays an important role in the conditions resulting from function $V_{2}$. In [4] it is chosen in the worst case, thus reducing the variation range of the parameters. In the following, we will be interested in changing the candidate storage function in order to find less conservative passivity conditions resulting in better tracking performance of the switching function $\bar{\gamma}$.

\section{B. Modifying the storage function}

In this section, we are interested in finding a novel candidate storage function that may allow us to conclude on system passivity in a less conservative way. To do so, let us fist modify the assumptions on (6) to replace $\alpha$ by a time-dependent matrix :

$$
r=\dot{e}_{p}+M^{-1} B e_{p}
$$

where $B \in \mathbb{R}^{m \times m}$ is a time-dependent diagonal matrix with bounded eigenvalues.

Lemma 1: Considering the filtered tracking error as defined in (20) with $M$ a constant diagonal matrix and $B$ a time-dependent diagonal matrix with bounded eigenvalues. If $r$ is bounded then $e_{p}, \dot{e}_{p}$ are bounded and that if $r \rightarrow 0$ then $e_{p}, \dot{e}_{p} \rightarrow 0$ (see Appendix A for the proof).

In this case, using the same strategy as previously in equation (9) yields

$$
\begin{array}{r}
\int_{0}^{t} r^{T} f_{e x t} d \tau=\int_{0}^{t}\left\{r^{T} M \dot{r}+e_{p}^{T} C \dot{e}_{p}+\frac{1}{2} e_{p}^{T} \dot{C} e_{p}\right\} d \tau \\
+\int_{0}^{t}\left\{\dot{e}_{p}^{T}(D-B) \dot{e}_{p}+e_{p}^{T}\left(B M^{-1}(K-\dot{B})-\frac{1}{2} \dot{C}\right) e_{p}\right. \\
\left.+e_{p}^{T}\left(K+B M^{-1}(D-B)-\dot{B}-C\right) \dot{e}_{p}\right\} d \tau
\end{array}
$$

that is

$$
\int_{0}^{t} r^{T} f_{e x t} d \tau=V_{3}(t)-V_{3}(0)+\int_{0}^{t} W_{3} d \tau
$$

with

$$
V_{3}=\frac{1}{2}\left[\begin{array}{c}
\dot{e}_{p} \\
e_{p}
\end{array}\right]^{T}\left[\begin{array}{cc}
M & B \\
B & C+B M^{-1} B
\end{array}\right]\left[\begin{array}{l}
\dot{e}_{p} \\
e_{p}
\end{array}\right]
$$

and

$$
\begin{array}{r}
W_{3}=\dot{e}_{p}^{T}(D-B) \dot{e}_{p}+e_{p}^{T}\left(B M^{-1}(K-\dot{B})-\frac{1}{2} \dot{C}\right) e_{p} \\
+e_{p}^{T}\left(K+B M^{-1}(D-B)-\dot{B}-C\right) \dot{e}_{p}
\end{array}
$$

The choice $C=K+B M^{-1}(D-B)-\dot{B}$ allows canceling out the cross-terms. According to equation (24), the positivity of $V_{3}$ is obtained as a consequence of Schur lemma [22] and the condition

$$
0 \leq C
$$

The positivity of $W_{3}$ is obtained if the following novel passivity conditions hold

$$
\begin{aligned}
& 0 \leq D-B \\
& 0 \leq B M^{-1}(K-\dot{B})-\frac{1}{2} \dot{C}
\end{aligned}
$$


It is worth noticing that the storage function $V_{2}$ of equation (10), initially defined by [4], is a special case of $V_{3}$ defined in equation (23) with $B=\alpha M, C=K+\alpha D$ and $\alpha$ constant. The analogy between the scalar $\alpha$ in equation (6) and the matrix $D M^{-1}$ in equation (20), and then between $\alpha M$ and $D$ suggests that the candidate storage function could be modified to limit the conservatism of the worst-case choice for $\alpha$. This leads to set $B=D$ and $C=K-\dot{D}$. A fourth storage function is then defined, such that

$$
V_{4}=\frac{1}{2}\left[\begin{array}{c}
\dot{e}_{p} \\
e_{p}
\end{array}\right]^{T}\left[\begin{array}{cc}
M & D \\
D & K+D M^{-1} D-\dot{D}
\end{array}\right]\left[\begin{array}{l}
\dot{e}_{p} \\
e_{p}
\end{array}\right]
$$

and the resulting passivity conditions are deduced from equations (25) to (27)

$$
\begin{aligned}
& 0 \leq K-\dot{D} \\
& 0 \leq 2 D M^{-1}(K-\dot{D})-\dot{K}+\ddot{D}
\end{aligned}
$$

As previously, all matrices are chosen diagonal and the problem can be decoupled, thus (30) is equivalent to

$$
\dot{k}_{i}+\frac{2}{m_{i}} d_{i} \dot{d}_{i}-\ddot{d}_{i}-\frac{2}{m_{i}} k_{i} d_{i} \leq 0
$$

resulting in

$$
-a_{1 i} \ddot{\gamma}_{i}+a_{2 i} \dot{\gamma}_{i}+a_{3 i} \gamma_{i} \dot{\gamma}_{i}-a_{4 i} \gamma_{i}-a_{5 i} \gamma_{i}^{2}-a_{6 i} \leq 0
$$

with

$$
\begin{aligned}
a_{1 i} & =\delta d_{i} & a_{2 i} & =\delta k_{i}+\frac{2}{m_{i}} d_{0 i} \delta d_{i} \\
a_{3 i} & =\frac{2}{m_{i}} \delta d_{i}^{2} & a_{4 i} & =\frac{2}{m_{i}}\left(d_{0 i} \delta k_{i}+k_{0 i} \delta d_{i}\right) \\
a_{5 i} & =\frac{2}{m_{i}} \delta k_{i} \delta d_{i} & a_{6 i} & =\frac{2}{m_{i}} k_{0 i} d_{0 i}
\end{aligned}
$$

which are all positive since $\delta K$ and $\delta D$ are positive. Then, as $a_{2 i}>0$ and $\gamma_{i}$ is such that $0 \leq \gamma_{i} \leq 1, \forall \gamma_{i}, a_{2 i}+a_{3 i} \gamma_{i}>0$. This allows to write the passivity condition

$$
\dot{\gamma}_{i} \leq \frac{a_{1 i} \ddot{\gamma}_{i}+a_{4 i} \gamma_{i}+a_{5 i} \gamma_{i}^{2}+a_{6 i}}{a_{2 i}+a_{3 i} \gamma_{i}} \triangleq h_{1 i}\left(\ddot{\gamma}_{i}, \gamma_{i}\right)
$$

Additionally, in the case where $\delta d_{i} \neq 0$, equation (29) gives a second passivity condition for $\dot{\gamma}_{i}$ such that

$$
\dot{\gamma}_{i} \leq \frac{\delta k_{i}}{\delta d_{i}} \gamma_{i}+\frac{k_{0 i}}{\delta d_{i}} \triangleq h_{2 i}\left(\gamma_{i}\right)
$$

Using passivity conditions (32) and (33), a new passivity filter similar to (19) could be defined such that

$$
\dot{\gamma}_{i}=\min \left(h_{1 i}\left(\ddot{\gamma}_{i}, \gamma_{i}\right), h_{2 i}\left(\gamma_{i}\right), \beta\left(\bar{\gamma}_{i}-\gamma_{i}\right)\right)
$$

Condition (32) is however not well suited for practical implementation as it requires derivating $\dot{\gamma}_{i}$, which is the output of a min-switch and therefore can be discontinuous. For this reason, we choose to use a more conservative but practically implementable constraint, such that

$$
\dot{\gamma}_{i} \leq \frac{a_{4 i} \gamma_{i}+a_{5 i} \gamma_{i}^{2}+a_{6 i}}{a_{2 i}+a_{3 i} \gamma_{i}} \triangleq h_{3 i}\left(\gamma_{i}\right)
$$

In fact, when the constraint is active, we have $\dot{\gamma}_{i} \geq 0$ and (35) becomes an equality. In this case it can be shown that

$$
\ddot{\gamma}_{i}=\frac{2 a_{5} a_{2} \gamma_{i}+a_{5} a_{3} \gamma_{i}^{2}+\left(a_{2} a_{4}-a_{3} a_{6}\right)}{\left(a_{2}+a_{3} \gamma_{i}\right)^{2}} \dot{\gamma}_{i}
$$

and

$$
a_{2} a_{4}-a_{3} a_{6}=\frac{4}{m_{i}^{2}} \delta k_{i}\left(\frac{m_{i}}{2} d_{0 i}+\delta d_{i} k_{0 i}+d_{0 i}^{2} \delta d_{i}\right) \geq 0
$$

resulting in $\ddot{\gamma}_{i} \geq 0$. This leads to the fact that if $\dot{\gamma}_{i}$ respects the constraint given by equation (35), then it also respects the passivity condition (32). Finally, the proposed passivity filter resulting from conditions (33) and (35) is defined by

$$
\dot{\gamma}_{i}=\min \left(h_{2 i}\left(\gamma_{i}\right), h_{3 i}\left(\gamma_{i}\right), \beta\left(\bar{\gamma}_{i}-\gamma_{i}\right)\right)
$$

\section{VALIDATION}

\section{A. Simulations}

In order to illustrate the advantages of the proposed approach, simulations analyzing passivity properties on a onedimensional mass-spring-damper system were performed. The simulated system has a constant mass $m=10 \mathrm{~kg}$, a stiffness varying between $k_{0}=2 \mathrm{~N} / \mathrm{m}$ and $k_{1}=22 \mathrm{~N} / \mathrm{m}$ and a damping ratio of 0.1 . The considered reference trajectory was set such that $p_{r}=10 \sin 0.1 t$. The stiffness is expected to vary according to the switching function $\bar{\gamma}$ illustrated in Fig. 1 in dashed gray. The passivity filters (19) and (36), associated to storage functions $V_{2}$ and $V_{4}$, respectively, are tuned with the parameter $\beta=10$. The resulting switching functions $\gamma$ are represented in Fig. 1. One can see that even

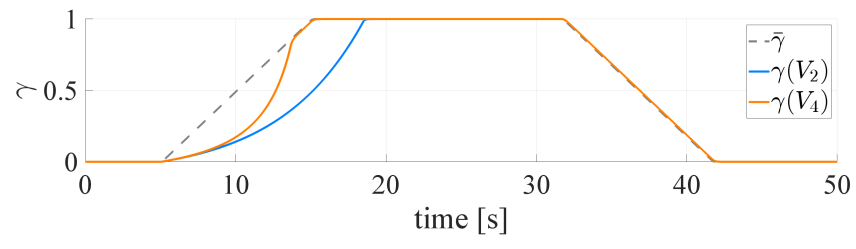

Fig. 1. Switching variable $\gamma$ resulting from filter with conditions from $V_{2}$ and $V_{4}$ and its reference.

if the outputs of both filters first evolve similarly, the output of filter (36) converges much more rapidly to the reference switching variable. This illustrates the fact that the conditions given by $V_{4}$ are less conservative than those given by $V_{2}$. Note that no external force is applied to the system, and in this case passivity is guaranteed if the derivative of the storage (Lyapunov-like) function is negative. As shown in Fig. 2, when comparing the evolution of $\dot{V}_{1}, \dot{V}_{2}$ and $\dot{V}_{4}$ in the case where $\gamma$ is calculated using passivity condition (19) (based on $V_{2}$ ), one can see that in contrary to function $V_{1}$ which is too conservative, $V_{2}$ and $V_{4}$ guarantee passivity. On the other hand, using passivity condition (36) (based on $V_{4}$ ), one can see in Fig. 3 that functions $V_{1}$ and $V_{2}$ cannot guarantee passivity, whereas $V_{4}$ is capable of doing so. 


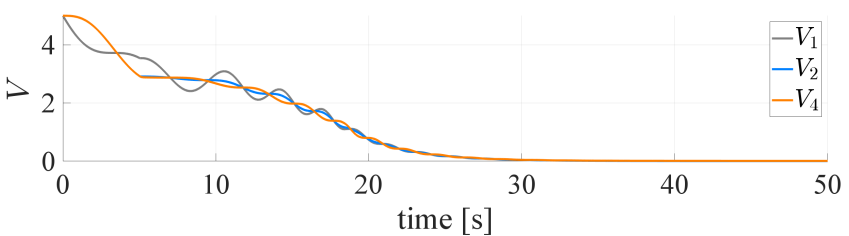

(a) Storage functions

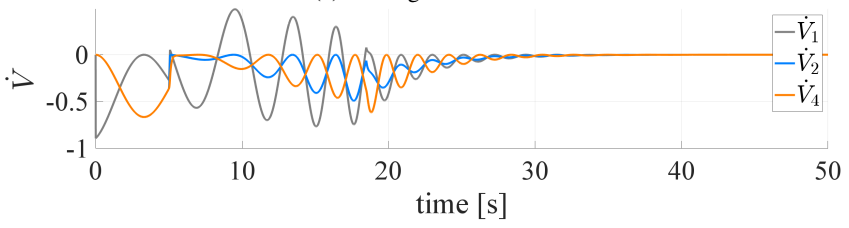

(b) Derivative of storage functions.

Fig. 2. Time evolution of storage functions for $\gamma$ calculated to respect passivity constraints (19) (based on $V_{2}$ ).

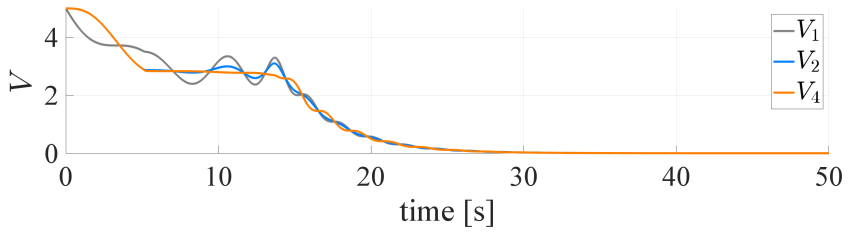

(a) Storage functions.

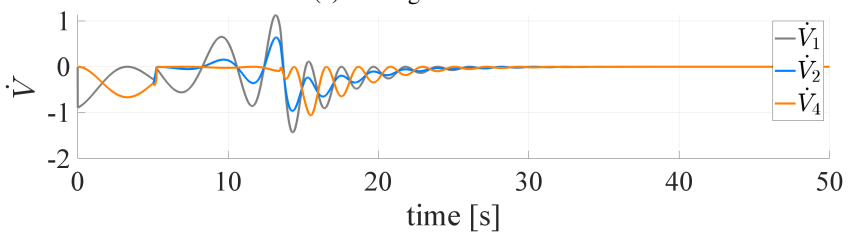

(b) Derivative of storage functions.

Fig. 3. Time evolution of storage functions for $\gamma$ calculated to respect passivity constraints (36) (based on $V_{4}$ ).

\section{B. Experiment}

The passivity filter (36) was then tested experimentally in a needle insertion task, inspired by the scenario described in [15]. A 16G medical needle was placed in a needle holder, mounted at the end-effector of a KUKA iiwa 14 robot, equipped with an ATI-Mini40 force-torque sensor (Fig. 4).

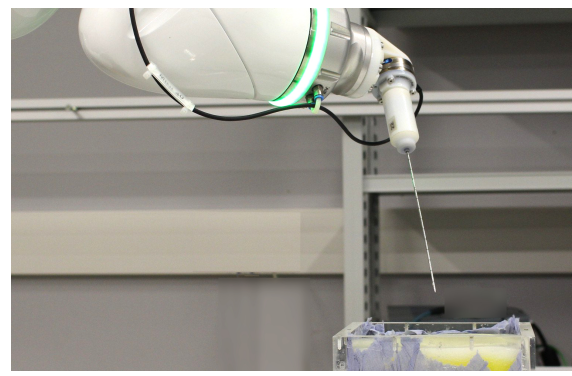

Fig. 4. Experimental setup.

The needle punctured a 4-layer silicon phantom, whose layers imitate skin, fat, muscle and liver, respectively. The muscle is the stiffest layer, followed by skin, fat and liver. Generally, during needle insertion procedures, no rotations of the needle are desired, thus we choose to change impedance profiles only in translation. The impedance parameters are chosen such that all inertial terms $m_{i}=5 \mathrm{~kg}$ and the damping ratio is set to be 1 . The desired stiffness varies with the depth in the phantom such that $\bar{k}_{i}=3000,3500,4000 \mathrm{~N} / \mathrm{m}$ for fat/liver, skin and muscle, respectively. Outside of the phantom, the system starts insertion with $\bar{k}_{i}=1000 \mathrm{~N} / \mathrm{m}$ and extracts the needle with $\bar{k}_{i}=3000 \mathrm{~N} / \mathrm{m}$. The filter parameter is $\beta=50$. The VIC algorithm implemented in $\mathrm{C}++$ generates torque commands fed to the system at $500 \mathrm{~Hz}$, using KUKA's Fast Robot Interface protocol.

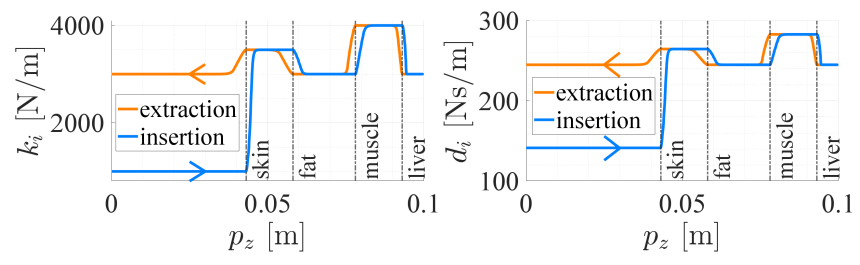

(a) Stiffness and damping profiles for needle insertion and extraction.

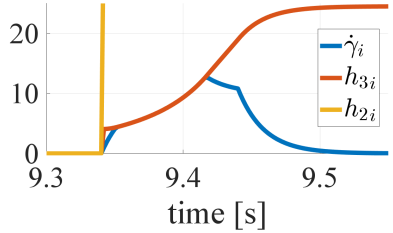

(b) Passivity constraints and $\dot{\gamma}_{i}$ during stiffness increase. Condition $h_{2 i}$ increases to high values and was cut-out of the scope for readability.

Fig. 5. Impedance profile and evolution of $\dot{\gamma}_{i}$.

The effective stiffness and damping $\left(k_{i}\right.$ and $\left.d_{i}\right)$, generated by the passivity filter are shown on Fig 5a. The influence of the passivity filter on the impedance profile can be observed when the needle punctures the skin, at $t=9.35 \mathrm{~s}$, and the stiffness is increased from 1000 to $3500 \mathrm{~N} / \mathrm{m}$. In this case, the velocity of the switching variable $\gamma_{i}$ increases freely until it meets the constraint $h_{3 i}$ imposed by the passivity filter (36) (Fig 5b) that shapes its further increase rate. This allows an important stiffness increase while guaranteeing passivity.

\section{CONCLUSIONS AND PERSPECTIVES}

In this paper we propose a design method for filters that permit the tracking of impedance profiles for variable impedance control, while guaranteeing passivity. Two filters are derived based on two candidate storage functions. The proposed passivity filters were validated in simulation and in an experimental setup featuring a KUKA iiwa collaborative robot executing a needle insertion task.

Future work aims at expanding the proposed filter design with other passivity conditions in search of even less conservative passivity guarantees. Also, it can be investigated how unusual impedance profile variations, such as increasing stiffness while decreasing damping, impact on the passivity guarantees and the design of the passivity filters.

\section{ACKNOWLEDGMENT}

This work was supported by the French government research program Investissements d'Avenir under the Robotex Equipment of Excellence and Labex CAMI (ANR-10EQPX- 44 and ANR-11-LABX-0004) and by the IDEX of the University of Strasbourg. 


\section{APPENDIX}

\section{A. Proof of Lemma 1.}

As the matrices $M$ and $B$ are chosen to be diagonal, the problem can be decoupled accordingly to the diagonal terms. Hence $\forall i \in\{1,2, \ldots, n\}$ :

1) if $r_{i}$ is bounded then $e_{p_{i}}, \dot{e}_{p i}$ are bounded: the solution of the differential equation

$$
r_{i}=\dot{e}_{p i}+\frac{b_{i}}{m_{i}} e_{p_{i}}
$$

is given by

$$
e_{p_{i}}=e^{-\int_{0}^{t} \frac{b_{i}(\tau)}{m_{i}} d \tau} e_{p_{i}}(0)+\int_{0}^{t} e^{-\int_{\tau}^{t} \frac{b_{i}(\sigma)}{m_{i}} d \sigma} r_{i}(\tau) d \tau
$$

As $b_{i} \in \mathbb{R}^{+}$is considered to be bounded such that $\underline{b}_{i} \leq b_{i} \leq \bar{b}_{i}$, (38) can be upper bounded by

$$
\begin{aligned}
\left|e_{p_{i}}\right| & \leq\left|e_{p_{i}}(0)\right|+\int_{0}^{t} e^{-\frac{\underline{b}_{i}}{m_{i}}(t-\tau)}\left|r_{i}(\tau)\right| d \tau \\
& \leq\left|e_{p_{i}}(0)\right|+\sup _{t}\left(\left|r_{i}(\tau)\right|\right) \int_{0}^{t} e^{-\frac{b_{i}}{m_{i}}(t-\tau)} d \tau \\
& \leq\left|e_{p_{i}}(0)\right|+\sup _{t}\left(\left|r_{i}(\tau)\right|\right) \frac{m_{i}}{\underline{b}_{i}}\left(1-e^{-\frac{\underline{b}_{i}}{m_{i}} t}\right)
\end{aligned}
$$

what shows that if $r_{i}$ is bounded then $e_{p_{i}}$ is bounded. Additionally, using (37) one get that

$$
\left|\dot{e}_{p i}\right| \leq \frac{\bar{b}_{i}}{m_{i}}\left|e_{p_{i}}\right|+\left|r_{i}(t)\right|
$$

that can be written using (39)

$$
\left|\dot{e}_{p i}\right| \leq \frac{\bar{b}_{i}}{m_{i}}\left|e_{p_{i}}(0)\right|+\sup _{t}\left(\left|r_{i}(\tau)\right|\right) \frac{\bar{b}_{i}}{\underline{b}_{i}}\left(1-e^{-\frac{\underline{b}_{i}}{m_{i}} t}\right)+\left|r_{i}(t)\right|
$$

what shows that if $r_{i}$ is bounded then $\dot{e}_{p i}$ is bounded.

2) if $r_{i} \rightarrow 0$ then $e_{p_{i}}, \dot{e}_{p i} \rightarrow 0$ : Considering the 2-norm for a scalar function $f \in \mathbb{R}$ defined by $\|f\|_{2}=\sqrt{\int_{0}^{\infty} f^{2}(\tau) d \tau}$, one can show that $\left\|e_{p_{i}}\right\|_{2}$ is bounded. Hence

$$
\begin{aligned}
\left\|e_{p_{i}}\right\|_{2} & =\left\|e^{-\frac{b_{i}}{m_{i}} t} e_{p_{i}}(0)+\int_{0}^{t} e^{-\int_{\tau}^{t} \frac{b_{i}(\sigma)}{m_{i}} d \sigma} r_{i}(\tau) d \tau\right\|_{2} \\
& \leq\left\|e^{-\frac{\underline{b}_{i}}{m_{i}} t} e_{p_{i}}(0)\right\|_{2}+\left\|\int_{0}^{t} e^{-\frac{\underline{b}_{i}}{m_{i}}(t-\tau)} r_{i}(\tau) d \tau\right\|_{2}
\end{aligned}
$$

as $\frac{b_{i}}{m_{i}}$ is constant, following the same steps of the proof of Lemma 1.6 in [21], one can show that $\left\|e^{-\frac{\underline{b}_{i}}{m_{i}} t} e_{p_{i}}(0)\right\|_{2}$ is bounded and that $\left\|\int_{0}^{t} e^{-\frac{\underline{b}_{i}}{m_{i}}(t-\tau)} r_{i}(\tau) d \tau\right\|_{2}$ is bounded as $\left\|r_{i}\right\|_{2}$ is bounded. This shows that $\left\|e_{p_{i}}\right\|_{2}$ is bounded. Using Corollary 1.1 from [21], as $e_{p_{i}}, \dot{e}_{p i}$ and $\left\|e_{p_{i}}\right\|_{2}$ are bounded, then $\lim _{t \rightarrow \infty} e_{p_{i}}=0$. Finally, from (40) with $\lim _{t \rightarrow \infty} e_{p_{i}}=0$ and $\lim _{t \rightarrow \infty} r_{i}=0$, one can conclude that $\lim _{t \rightarrow \infty} \dot{e}_{p i}=0$.

\section{REFERENCES}

[1] N. Hogan, "Impedance control: An approach to manipulation," Journal of Dynamlc Systems, Measurement, and Contral, vol. 107, pp. 1-7, Mar 1985.

[2] J. E. Colgate and N. Hogan, "Robust control of dynamically interacting systems," International Journal of Control, vol. 48, no. 1, pp. 65-88, Jul 1988 .

[3] N. Hogan, "On the stability of manipulators performing contact tasks," IEEE Journal on Robotics and Automation, vol. 4, no. 6, pp. 677-686, Dec 1988

[4] K. Kronander and A. Billard, "Stability considerations for variable impedance control," IEEE Transactions on Robotics, vol. 32, no. 5, pp. 1298-1305, Oct 2016.

[5] R. Ikeura and H. Inooka, "Variable impedance control of a robot for cooperation with a human," in IEEE International Conference on Robotics and Automation (ICRA), vol. 3, 1995, pp. 3097-3102.

[6] D. Braun, M. Howard, and S. Vijayakumar, "Optimal variable stiffness control: formulation and application to explosive movement tasks," Autonomous Robots, vol. 33, no. 3, pp. 237-253, Oct 2012.

[7] M. Garabini, A. Passaglia, F. Belo, P. Salaris, and A. Bicchi, "Optimality principles in variable stiffness control: The VSA hammer," in IEEE/RSJ International Conference on Intelligent Robots and Systems, Sep 2011, pp. 3770-3775.

[8] J. R. Medina, D. Sieber, and S. Hirche, "Risk-sensitive interaction control in uncertain manipulation tasks," in IEEE International Conference on Robotics and Automation (ICRA), May 2013, pp. 502-507.

[9] S. Haddadin, M. Weis, S. Wolf, and A. Albu-Schaeffer, "Optimal control for maximizing link velocity of robotic variable stiffness joints," IFAC Proceedings Volumes, vol. 44, no. 1, pp. 6863-6871, Jan 2011.

[10] J. Buchli, F. Stulp, E. Theodorou, and S. Schaal, "Learning variable impedance control," The International Journal of Robotics Research, vol. 30, no. 7, pp. 820-833, Jun 2011.

[11] T. Tsumugiwa, R. Yokogawa, and K. Hara, "Variable impedance control based on estimation of human arm stiffness for human-robot cooperative calligraphic task," in IEEE International Conference on Robotics and Automation (ICRA), vol. 1, 2002, pp. 644-650.

[12] S. Grafakos, F. Dimeas, and N. Aspragathos, "Variable admittance control in pHRI using EMG-based arm muscles co-activation," in IEEE International Conference on Systems, Man, and Cybernetics (SMC), Oct 2016, pp. 1900-1905.

[13] F. Ficuciello, L. Villani, and B. Siciliano, "Variable impedance control of redundant manipulators for intuitive human-robot physical interaction," IEEE Transactions on Robotics, vol. 31, no. 4, pp. 850-863, Aug 2015.

[14] K. Lee and M. Buss, "Force tracking impedance control with variable target stiffness," IFAC Proceedings Volumes, vol. 41, no. 2, pp. 67516756,2008

[15] F. Ferraguti, C. Secchi, and C. Fantuzzi, "A tank-based approach to impedance control with variable stiffness," in IEEE International Conference on Robotics and Automation (ICRA), 2013, pp. 49484953.

[16] F. Ferraguti, N. Preda, A. Manurung, M. Bonfe, O. Lambercy, R. Gassert, R. Muradore, P. Fiorini, and C. Secchi, "An energy tankbased interactive control architecture for autonomous and teleoperated robotic surgery," IEEE Transactions on Robotics, vol. 31, no. 5, pp 1073-1088, Oct 2015 .

[17] C. Schindlbeck and S. Haddadin, "Unified passivity-based cartesian force/impedance control for rigid and flexible joint robots via taskenergy tanks," in IEEE International Conference on Robotics and Automation (ICRA), May 2015, pp. 440-447.

[18] E. Lutscher, E. C. Dean-Leon, and G. Cheng, "Hierarchical force and positioning task specification for indirect force controlled robots,' IEEE Transactions on Robotics, vol. 34, no. 1, pp. 280-286, Feb 2018.

[19] B. Siciliano and O. Khatib, Springer Handbook of Robotics. Springer International Publishing, 2016.

[20] A. Behal, W. Dixon, D. M. Dawson, and B. Xian, Lyapunov-Based Control of Robotic Systems. CRC Press, 2009.

[21] D. M. Dawson, Nonlinear Control of Electric Machinery. CRC Press, 2019

[22] S. P. Boyd, Linear matrix inequalities in system and control theory, ser. SIAM studies in applied mathematics. Society for Industrial and Applied Mathematics, 1994. 